\title{
Mechanical and Aspiration Thrombectomy in a 2-day-old Neonate with Perinatal Stroke
}

\author{
Kamil Zeleňák ${ }^{1,2}$ (D) Katarina Matasova jr. ${ }^{2,3}$ (D) Anna Bobulova ${ }^{1,2}$ (D) Katarina Matasova $^{2,3}$ (D)
}

Received: 8 May 2021 / Accepted: 14 September 2021 / Published online: 13 October 2021

(c) Springer-Verlag GmbH Germany, part of Springer Nature 2021

\section{Introduction}

Perinatal arterial ischemic stroke (PAIS) is a most common subtype of perinatal stroke occurring between the 20th week of pregnancy and 28th day of postnatal life. It is caused by vascular occlusion in an arterial territory secondary to arterial thrombosis or embolization. In most cases there is the involvement of the left middle cerebral artery (MCA) manifested by convulsions. They are usually focal, brief, and contralateral to the affected hemisphere and occur $12-72 \mathrm{~h}$ after delivery. The gold standard of diagnosis is magnetic resonance imaging (MRI) including diffusionweighted imaging (DWI) that shows the focal brain involvement in the territory of the affected artery $[1,2]$.

Although PAIS affects 1 in 2300-5000 newborns, there is no clear recommendation for the management in the literature [1]. The treatment is usually conservative. In addition to standard anticonvulsant therapy, the neuroprotective effects of erythropoietin and stem cells are also considered. The use of anticoagulants is still discussed because of the minimal risk of recurrent stroke after PAIS [3]. The use of

Kamil Zeleňák

kamil.zelenak@uniba.sk

Katarina Matasova jr.

matasova.katka@gmail.com

Anna Bobulova

abobulova@gmail.com

Katarina Matasova

matasova.katarina@gmail.com

1 Department of Radiology, Jessenius Faculty of Medicine in Martin, Comenius University in Bratislava, Mala Hora 4, 036 01 Martin, Slovakia

2 University Hospital Martin, Kollarova 2, 03659 Martin, Slovakia

3 Department of Neonatology, Jessenius Faculty of Medicine in Martin, Comenius University in Bratislava, Mala Hora 4, 036 01 Martin, Slovakia hyperacute stroke therapy (thrombolysis, mechanical and aspiration thrombectomy) has not been reported in neonatal age $[1,3]$.

We present a case of a neonate with occlusion of the left MCA successfully treated by combination of mechanical and aspiration thrombectomy at the age of 2 days (in October 2020).

\section{Case Report}

The eutrophic male neonate was born at the 41 st week of an uncomplicated pregnancy. The immediate postpartum adaptation was appropriate and the postnatal life was initially uneventful.

At the age of $24 \mathrm{~h}$, a focal aware motor seizure (the right upper and lower limbs) lasting for $1 \mathrm{~min}$ was observed. In the next $16 \mathrm{~h}$ a focal to bilateral tonic-clonic seizure reoccurred. Right upper limb hypotonia and weak Moro reflex on the right side persisted after the seizure. Subsequently, brain ultrasonography was performed (with a normal finding) and anticonvulsant therapy (phenobarbital) was started. The child was referred to a specialized centre with availability of endovascular therapy. Noncontrast-enhanced computed tomography (CT), CT angiography, CT perfusion (CTP) and MRI including DWI confirmed acute ischemic stroke affecting the left frontal and temporal lobes due to the cranial M2 branch of the left MCA occlusion (Figs. 1 and $2 \mathrm{~d}$ ).

After multidisciplinary consensus and parental consent, endovascular treatment was performed with the patient under general anesthesia. The exact time of the stroke onset was difficult to establish, we assumed an onset-to-groin time of about $8 \mathrm{~h}$. The right femoral access was used. Initial puncture was performed with $3 \mathrm{~F}$ micropuncture set, replaced by a $5 \mathrm{~F}$ introducer. Aspiration catheter Sofia EX $105 \mathrm{~cm}$ STR (MicroVention, Tustin, CA, USA) 5F was placed into the M1 segment of the left MCA. Subsequently a microcatheter Excelsior SL-10/45 (Stryker Neurovascu- 
Fig. 1 a, b Noncontrast-enhanced CT: hypodensity of grey and white matter in the left frontal and temporal lobes. c Noncontrast-enhanced CT: dense artery sign corresponding to acute left MCA occlusion (arrow). d CT angiography: confirmed occlusion of the MCA (arrow). e CT perfusion maps presenting elevated $\mathrm{CBV}$ (cerebral blood volume), moderately reduced CBF (cerebral blood flow) and prolonged MTT (mean transit time) in affected brain tissue suggesting penumbra cranial M2 branch of the left
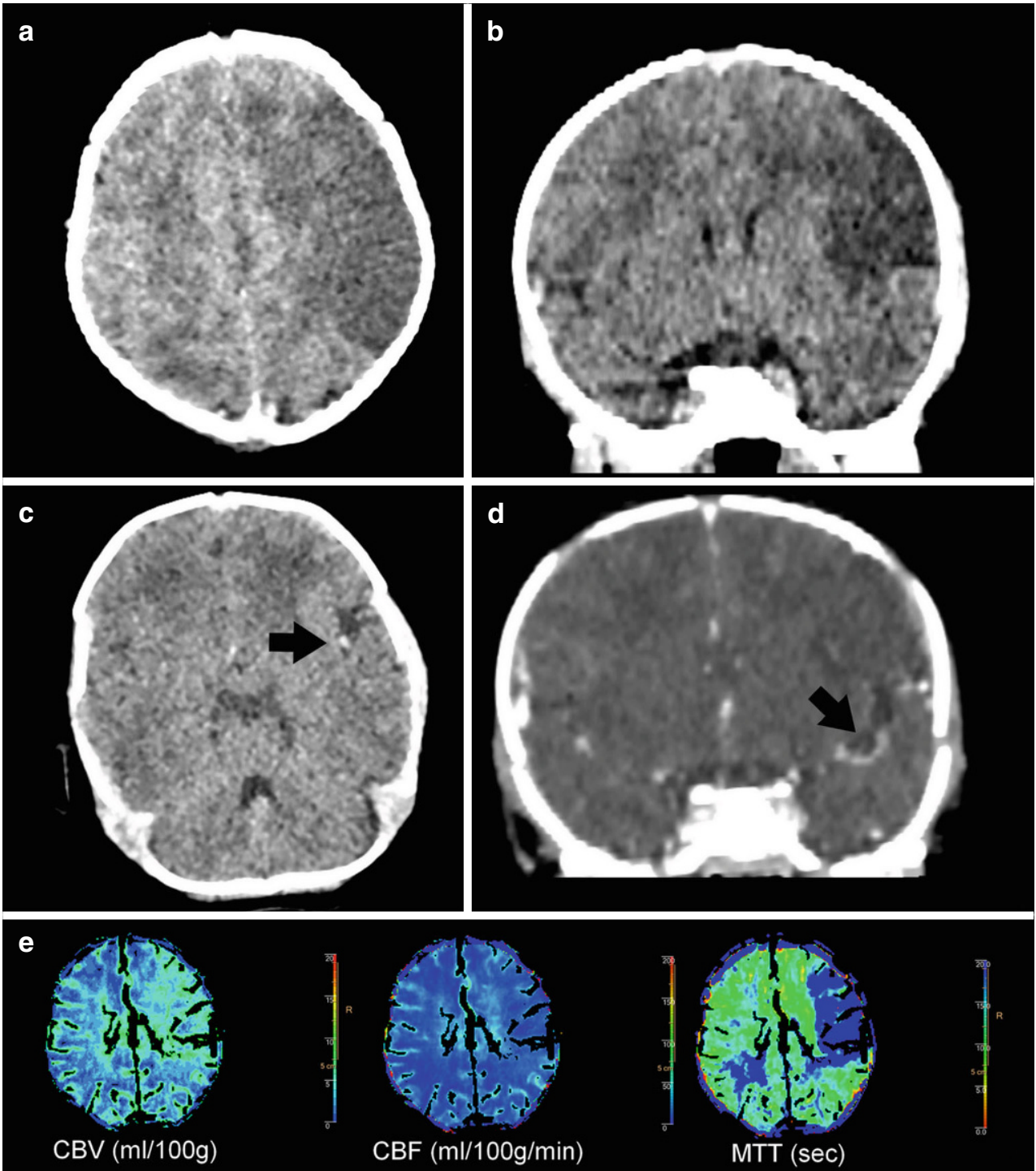

lar, Fremont, CA, USA) was navigated into the occluded branch with Hybrid 0.008 J (Balt, Montmorency, France). Thrombectomy device pRESET LITE $3 \times 20 \mathrm{~mm}$ (Phenox, Bochum, Germany) was used. The first pass effect and recanalization of the MCA (TICI-3 - Thrombolysis in cerebral infarction scale) was achieved (Fig. 2a-c) (time grointo-reperfusion was $40 \mathrm{~min}$ ).

Nimodipine was administered continuously $(15-30 \mu \mathrm{g} / \mathrm{kg} / \mathrm{h})$ for 12 days to prevent vasospasms after the procedure. No hemodynamic concerns occurred. A focal subarachnoid hemorrhage was confirmed the next day by imaging technique, it resolved spontaneously. Prophylactic treatment with low molecular weight heparin was initiated 9 days after the procedure. The muscle tone of the right extremities was normalized almost immediately after recanalization and the seizure did not reoccur. The child was discharged home at the age of 27 days without any motor deficits.
Follow-up MRI 3 months later displayed a pseudocystic transformation of the ischemia-damaged brain tissue, but smaller in size, compared to initial noncontrast-enhanced CT and DWI lesion (Fig. 2d-f). The reperfused M2 branch showed still patency (Fig. 2g). Psychomotor functions of the baby were age-appropriate without neurological deficits.

\section{Discussion}

The case of a PAIS manifested by focal seizures is presented in our case report. The use of CT and MRI imaging detected ischemia in the left MCA territory that is the most common localization of the infarction in PAIS $[1,2]$. With respect to a substantial volume of the ischemic lesion CTP was performed. Although we assumed the irreversible damage of the affected area, it corresponded to the penumbra in adults. Currently, no guidelines for the evaluation of 
Fig. 2 a Digital subtraction angiography (DSA): occlusion of the M2 branch of the left MCA (arrow). b DSA: placement of guidewire and microcatheter into the occluded branch. $\mathbf{c}$ DSA: complete recanalization in the Thrombolysis in cerebral infarction scale (TICI-3). d MRI: DWI before endovascular recanalization: hyperintense area of restricted diffusion in the left MCA territory. e , f Followup MRI 3 months later: T1weighted image: final ischemic brain tissue volume was smaller than the initial DWI lesion. g Follow-up time-of-flight MR angiography 3 months later shows the patency of recanalized M2 branch of the left MCA (arrow)
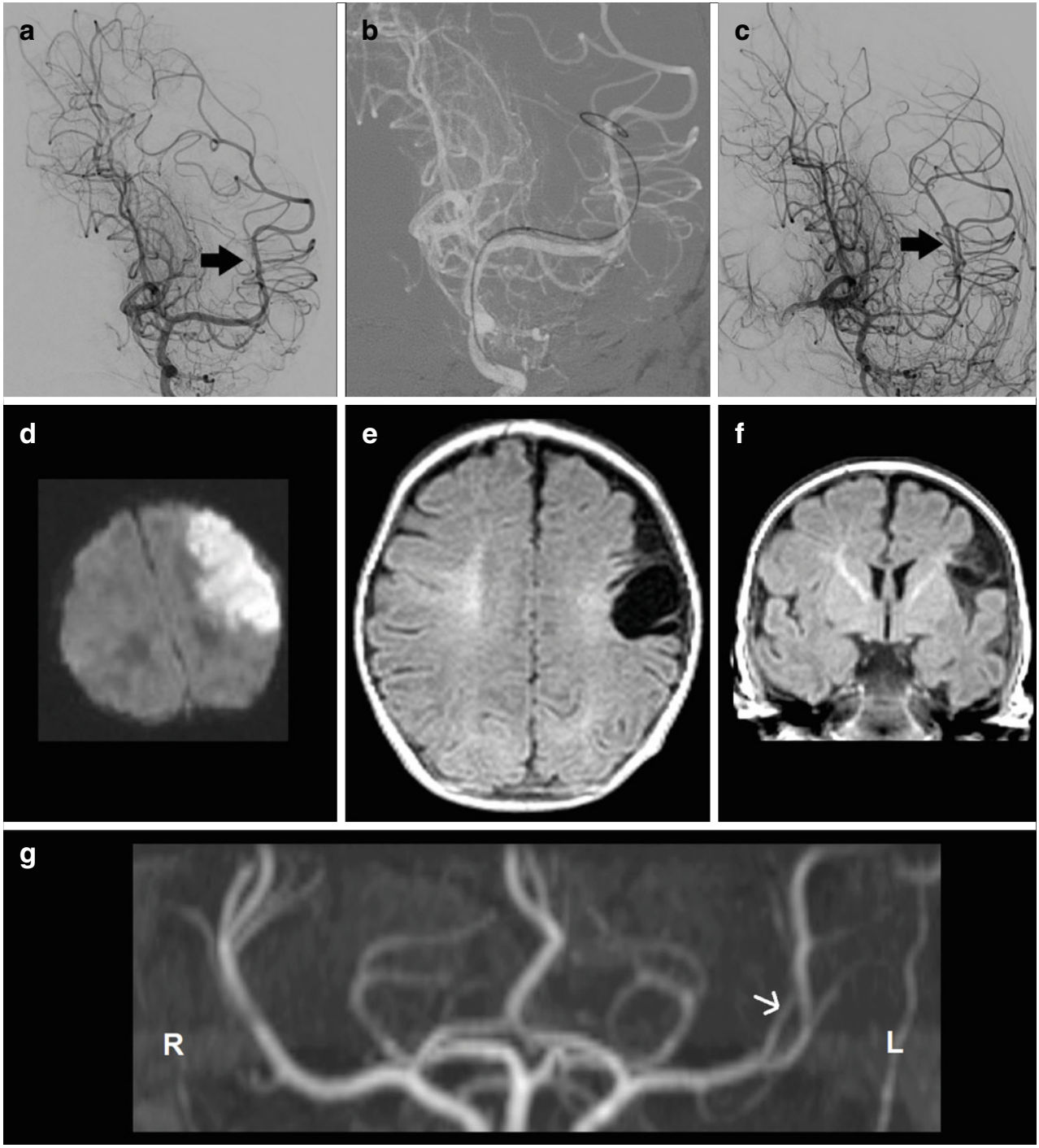

a neonatal brain CTP in emergency settings are available [4-6]. Based on the literature we hypothesize that immature vascularization could be the main reason for a different CTP imaging in the adult and neonatal populations. In our patient $\mathrm{CBV}$ in the affected area was not reduced but increased in a compensatory manner.

There is no evidence for hyperacute stroke therapies for neonates with an arterial occlusion [3] but a recent trial suggested the same safety profile of thrombectomy in children as in adults and supports clinicians' practice of off-label thrombectomy in childhood stroke [7]. The decision-making process was an ethical dilemma: we considered harmful effects of endovascular therapy, the large lesion volume and the fact the M2 occlusion seems to affect the superior trunk, which has important clinical relevance and opinion of parents as well. Due to the uncertainty of the irreversible brain damage and despite the commonly used conservative therapy, endovascular treatment was performed. A focal subarachnoid hemorrhage after the intervention was insignifi- cant and it is reported in $16.4 \%$ of adults after mechanical thrombectomy [8].

To the best of our knowledge this is the first reported case of endovascular complete recanalization of the MCA in a neonate. The neurological status improved significantly after the procedure, symmetry of reflexes and motor activity on both sides was observed. The final volume of ischemic area was smaller compared to the initial DWI lesion. A possible cause for smaller size of the defect than initially could be the compensatory increase in CBV.

The exact cause of the PAIS in our patient was not established. We assumed a placental pathology leading to embolization. The association between fetal vascular malperfusion (a spectrum of histologic placental abnormalities) and perinatal stroke is increasingly acknowledged [9]. To confirm the placental disorder, a histological evaluation of the placenta would be required [1, 2, 9]. Thrombophilic state in the mother was excluded and coagulation tests in the child were normal. 
In the context of the current pandemic situation, it is important to note that in acute coronavirus disease 2019 (COVID-19) there are histopathological signs of maternal and fetal malperfusion present in $100 \%$ and $40 \%$ of cases, respectively [10]. Based on these facts we can hypothesize an increasing incidence of PAIS. In adult patients with acute ischemic stroke improved clinical outcome 3 months after endovascular treatment was proven [11]. Our aim was to demonstrate feasibility of mechanical and aspiration thrombectomy in the management of the MCA occlusion in neonatal period improving the neurological outcome. The advances in technology used for thrombectomy in smaller-sized or medium-sized vessel occlusions in adults [12] make stroke interventions in neonatal patients possible.

\section{Conclusion}

The endovascular treatment in MCA occlusion is feasible in neonates and can lead to clinical improvement and reduction of the damaged brain tissue volume. It should be considered in the management of a neonate with PAIS.

Author Contribution K. Zelenak performed the procedure and Katarina Matasova supervised the management of the patient; A. Bobulova participated in diagnostic process and prepared the figures; The first draft of the manuscript was written by K. Matasova jr. All authors commented on the manuscript and contributed to the final version; Subsequently all authors read and approved the final version to be published.

\section{Declarations}

Conflict of interest K. Zeleňák, K. Matasova jr., A. Bobulova and $\mathrm{K}$. Matasova declare that they have no competing interests.

Ethical standards All procedures performed in studies involving human participants or on human tissue were in accordance with the ethical standards of the institutional and/or national research committee and with the 1975 Helsinki declaration and its later amendments or comparable ethical standards. The parents have been offered the opportunity to read the manuscript. Parents gave written informed consent regarding publishing data and images of their child.

\section{References}

1. Roach GD. Perinatal Arterial Ischemic Stroke. Neoreviews. 2020; 21:e741-8.

2. Dunbar M, Kirton A. Perinatal Stroke. Semin Pediatr Neurol. 2019;32:100767.

3. Ferriero DM, Fullerton HJ, Bernard TJ, Billinghurst L, Daniels SR, DeBaun MR, et al.; American Heart Association Stroke Council and Council on Cardiovascular and Stroke Nursing. Management of Stroke in Neonates and Children: A Scientific Statement From the American Heart Association/American Stroke Association. Stroke. 2019;50:e51-96.

4. Donahue MJ, Dlamini N, Bhatia A, Jordan LC. Neuroimaging Advances in Pediatric Stroke. Stroke. 2019;50:240-8.

5. Khalaf A, Iv M, Fullerton H, Wintermark M. Pediatric Stroke Imaging. Pediatr Neurol. 2018;86:5-18.

6. Proisy M, Mitra S, Uria-Avellana C, Sokolska M, Robertson NJ, Le Jeune F, Ferré JC. Brain Perfusion Imaging in Neonates: An Overview. AJNR Am J Neuroradiol. 2016;37:1766-73.

7. Sporns PB, Sträter R, Minnerup J, Wiendl H, Hanning U, Chapot R, et al. Feasibility, Safety, and Outcome of Endovascular Recanalization in Childhood Stroke: The Save ChildS Study. JAMA Neurol. 2020;77:25-34.

8. Maegerlein C, Prothmann S, Lucia KE, Zimmer C, Friedrich B, Kaesmacher J. Intraprocedural Thrombus Fragmentation During Interventional Stroke Treatment: A Comparison of Direct Thrombus Aspiration and Stent Retriever Thrombectomy. Cardiovasc Intervent Radiol. 2017;40:987-93.

9. Geraldo AF, Parodi A, Bertamino M, Buffelli F, Uccella S, Tortora D, et al. Perinatal Arterial Ischemic Stroke in Fetal Vascular Malperfusion: A Case Series and Literature Review. AJNR Am J Neuroradiol. 2020;41:2377-83.

10. Menter T, Mertz KD, Jiang S, Chen H, Monod C, Tzankov A, et al. Placental Pathology Findings during and after SARS-CoV-2 Infection: Features of Villitis and Malperfusion. Pathobiology. 2021;88:69-77.

11. Falk-Delgado A, Kuntze Söderqvist Å, Fransén J, Falk-Delgado A. Improved clinical outcome 3 months after endovascular treatment, including thrombectomy, in patients with acute ischemic stroke: a meta-analysis. J Neurointerv Surg. 2016;8:665-70.

12. Meyer L, Stracke CP, Jungi N, Wallocha M, Broocks G, Sporns PB, et al. Thrombectomy for Primary Distal Posterior Cerebral Artery Occlusion Stroke: The TOPMOST Study. JAMA Neurol. 2021;78: 434-44. 